\title{
Improved Bounds for the Excluded-Minor Approximation of Treedepth
}

\author{
Wojciech Czerwiński
}

Institute of Informatics, University of Warsaw, Poland

wczerwin@mimuw.edu.pl

Wojciech Nadara

Institute of Informatics, University of Warsaw, Poland

W.Nadara@mimuw.edu.pl

\section{Marcin Pilipczuk}

Institute of Informatics, University of Warsaw, Poland malcin@mimuw.edu.pl

\begin{abstract}
Treedepth, a more restrictive graph width parameter than treewidth and pathwidth, plays a major role in the theory of sparse graph classes. We show that there exists a constant $C$ such that for every integers $a, b \geq 2$ and a graph $G$, if the treedepth of $G$ is at least $C a b \log a$, then the treewidth of $G$ is at least $a$ or $G$ contains a subcubic (i.e., of maximum degree at most 3 ) tree of treedepth at least $b$ as a subgraph.

As a direct corollary, we obtain that every graph of treedepth $\Omega\left(k^{3} \log k\right)$ is either of treewidth at least $k$, contains a subdivision of full binary tree of depth $k$, or contains a path of length $2^{k}$. This improves the bound of $\Omega\left(k^{5} \log ^{2} k\right)$ of Kawarabayashi and Rossman [SODA 2018].

We also show an application for approximation algorithms of treedepth: given a graph $G$ of treedepth $k$ and treewidth $t$, one can in polynomial time compute a treedepth decomposition of $G$ of width $\mathcal{O}\left(k t \log ^{3 / 2} t\right)$. This improves upon a bound of $\mathcal{O}\left(k t^{2} \log t\right)$ stemming from a tradeoff between known results.

The main technical ingredient in our result is a proof that every tree of treedepth $d$ contains a subcubic subtree of treedepth at least $d \cdot \log _{3}((1+\sqrt{5}) / 2)$.
\end{abstract}

2012 ACM Subject Classification Mathematics of computing $\rightarrow$ Graph theory

Keywords and phrases treedepth, excluded minor

Digital Object Identifier 10.4230/LIPIcs.ESA.2019.34

Related Version A full version of the paper is available at https://arxiv.org/abs/1904.13077.

Funding This research is a part of a project that have received funding from the European Research Council (ERC) under the European Union's Horizon 2020 research and innovation programme Grant Agreement 714704.

\section{Introduction}

For an undirected graph $G$, the treedepth of $G$ is the minimum height of a rooted forest whose ancestor-descendant closure contains $G$ as a subgraph. Together with more widely known related width notions such as treewidth and pathwidth, it plays a major role in structural graph theory, in particular in the study of general sparse graph classes $[8,7,6]$.

An important property of treedepth is that it admits a number of equivalent definitions. Following the definition of treedepth above, a treedepth decomposition of a graph $G$ consists of a rooted forest $F$ and an injective mapping $f: V(G) \rightarrow V(F)$ such that for every $u v \in E(G)$ the vertices $f(u)$ and $f(v)$ are in ancestor-descendant relation in $F$. The width of a treedepth decomposition $(F, f)$ is the height of $F$ (the number of vertices on the longest leaf-to-root path in $F$ ) and the treedepth of $G$ is the minimum possible height of a treedepth decomposition

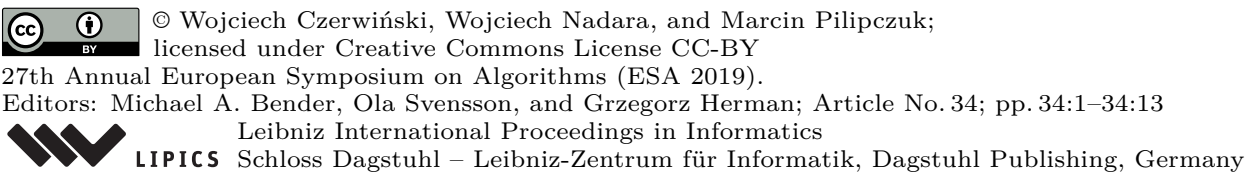


of $G$. A centered coloring of a graph $G$ is an assignment $\alpha: V(G) \rightarrow \mathbb{Z}$ such that for every connected subgraph $H$ of $G, \alpha$ has a center in $H$ : a vertex $v \in V(H)$ of unique color, i.e., $\alpha(v) \neq \alpha(w)$ for every $w \in V(H) \backslash\{v\}$. A vertex ranking of a graph $G$ is an assignment $\alpha: V(G) \rightarrow \mathbb{Z}$ such that in every connected subgraph $H$ of $G$ there is a unique vertex of maximum rank (value $\alpha(v)$ ). Clearly, each vertex ranking is a centered coloring. It turns out that the minimum number of colors (minimum size of the image of $\alpha$ ) needed for a centered coloring and for a vertex ranking are equal and equal to the treedepth of a graph [6].

While there are multiple examples of algorithmic usage of treedepth in the theory of sparse graphs $[7,8]$, our understanding of the complexity of computing minimum width treedepth decompositions is limited. For a graph $G$, let $\operatorname{td}(G)$ and $\operatorname{tw}(G)$ denote the treedepth and the treewidth of $G$, respectively. An algorithm of Reidl, Rossmanith, Villaamil, and Sikdar [9] computes exactly the treedepth of an input graph $G$ in time $2^{\mathcal{O}(\operatorname{td}(G) \cdot t)} n^{\mathcal{O}(1)}$, given a tree decomposition of $G$ of width $t$. Combined with the classic constant-factor approximation algorithm for treewidth that runs in $2^{\mathcal{O}(t w(G))} n^{\mathcal{O}(1)}$ time [10], one obtains an exact algorithm for treedepth running in time $2^{\mathcal{O}(\operatorname{td}(G) \operatorname{tw}(G))} n^{\mathcal{O}(1)}$. No faster exact algorithm is known.

For approximation algorithms, the following folklore lemma (presented with full proof in [4]) is very useful.

- Lemma 1. Given a graph $G$ and a tree decomposition $(T, \beta)$ of $G$ of maximum bag size $w$, one can in polynomial time compute a treedepth decomposition of $G$ of width at most $w \cdot \operatorname{td}(T)$.

Using Lemma 1, one can obtain an approximation algorithm for treedepth with a cheap tradeoff trick. ${ }^{1}$

- Lemma 2. Given a graph $G$, one can in polynomial time compute a treedepth decomposition of $G$ of width $\mathcal{O}\left(\operatorname{td}(G) \cdot \operatorname{tw}(G)^{2} \log \operatorname{tw}(G)\right)$.

Proof. Let $n=|V(G)|$. Using the polynomial-time approximation algorithm for treewidth [3], compute a tree decomposition $(T, \beta)$ of $G$ of width $t=\mathcal{O}(\operatorname{tw}(G) \sqrt{\log \operatorname{tw}(G)})$ and $\mathcal{O}(n)$ bags. For every integer $1 \leq k \leq(\log n) / t$, use the algorithm of [9] to check in polynomial time if the treedepth of $G$ is at most $k$. Note that if this is the case, the algorithm finds an optimal treedepth decomposition and we can conclude. Otherwise, we have $\log n \leq \operatorname{td}(G) \cdot t$ and we apply Lemma 1 to $G$ and $(T, \beta)$ obtaining a treedepth decomposition of $G$ of width

$$
\mathcal{O}(t \log n) \leq \mathcal{O}\left(\operatorname{td}(G) \cdot t^{2}\right) \leq \mathcal{O}\left(\operatorname{td}(G) \cdot \operatorname{tw}(G)^{2} \log \operatorname{tw}(G)\right) .
$$

Lemma 2 is the only polynomial approximation algorithm for treedepth running in polynomial time we are aware of.

A related topic to exact and approximation algorithms computing minimum-width treedepth decomposition is the study of obstructions to small treedepth. Dvořák, Giannopoulou, and Thilikos [2] proved that every minimal graph of treedepth $k$ has the number of vertices at most double-exponential in $k$. More recently, Kawarabayashi and Rossman showed an excluded-minor theorem for treedepth.

- Theorem 3 ([4]). There exists a universal constant $C$ such that for every integer $k$ every graph of treedepth at least $C k^{5} \log ^{2} k$ is either of treewidth at least $k$, contains a subdivision of a full binary tree of depth $k$ as a subgraph, or contains a path of length $2^{k}$.

1 This trick has been observed and communicated to us by Michał Pilipczuk. We thank Michał for allowing us to include it in this paper. 
While neither the results of [2] nor [4] have a direct application in the approximability of treedepth, these topics are tightly linked with each other and we expect that a finer understanding of treedepth obstructions is necessary to provide more efficient algorithms computing or approximating the treedepth of a graph.

\section{Our results}

Our main result is the following statement, improving upon the work of Kawarabayashi and Rossman [4].

- Theorem 4. Let $G$ be a graph of treewidth $\operatorname{tw}(G)$ and treedepth $\operatorname{td}(G)$. Then there exists a subcubic tree $H$ that is a subgraph of $G$ and is of treedepth $\Omega(\operatorname{td}(G) /(\operatorname{tw}(G) \log \operatorname{tw}(G)))$.

In other words, Theorem 4 states that there exists a constant $C$ such that for every graph $G$ and integers $a, b \geq 2$, if the treedepth of $G$ is at least $C a b \log a$, then the treewidth of $G$ is at least $a$ or $G$ contains a subcubic tree of treedepth $b$. Since every subcubic tree of treedepth $d$ contains either a simple path of length $2^{\Omega(\sqrt{d})}$ or a subdivision of a full binary tree of depth $\Omega(\sqrt{d})[4]$, we have the following corollary.

- Corollary 5. Let $G$ be a graph of treewidth $\operatorname{tw}(G)$ and treedepth $\operatorname{td}(G)$. Then for some

$$
h=\Omega(\sqrt{\operatorname{td}(G) /(\operatorname{tw}(G) \log \operatorname{tw}(G))})
$$

$G$ contains either a simple path of length $2^{h}$ or a subdivision of a full binary tree of depth $h$.

Consequently, there exists an absolute constant $C$ such that for every integer $k \geq 1$ and a graph $G$ of treedepth at least $C k^{3} \log k$, either

- $G$ has treewidth at least $k$,

- $G$ contains a subdivision of a full binary tree of depth $k$ as a subgraph, or

- $G$ contains a path of length $2^{k}$.

In other words, Corollary 5 improves the bound $k^{5} \log ^{2} k$ of Kawarabayashi and Rossman [4] to $k^{3} \log k$. We remark here that there are subcubic trees of treedepth $\Omega\left(h^{2}\right)$ that contains neither a path of length $2^{h}$ nor a subdivision of a full binary tree of depth $h,{ }^{2}$ and thus the quadratic loss between the statements of Theorem 4 and Corollary 5 is necessary.

Inside the proof of Theorem 4 we make use of the following lemma that may be of independent interest. This lemma is the main technical improvement upon the work of Kawarabayashi and Rossman [4].

- Lemma 6. Every tree of treedepth d contains a subcubic subtree of treedepth at least $\frac{\log ((1+\sqrt{5}) / 2)}{\log (3)} d$.

Furthermore, such a subtree can be found in polynomial time.

The techniques developed to prove Theorem 4 have some implications on the approximability of treedepth. We improve upon Lemma 2 as follows.

- Theorem 7. Given a graph $G$, one can in polynomial time compute a treedepth decomposition of $G$ of width $\mathcal{O}\left(\operatorname{td}(G) \cdot \operatorname{tw}(G) \log ^{3 / 2} \operatorname{tw}(G)\right)$.

\footnotetext{
${ }^{2}$ It is straightforward to deduce such an example from the proof of [4]. We provide such an example in Section 6.
} 
The result of Kawarabayashi and Rossman [4] has been also an important ingredient in the study of linear colorings [5]. A coloring $\alpha: V(G) \rightarrow \mathbb{Z}$ of a graph $G$ is a linear coloring if for every (not necessarily induced) path $P$ in $G$ there exists a vertex $v \in V(P)$ of unique color $\alpha(v)$ on $P$. Clearly, each centered coloring is a linear coloring, but the minimum number of colors needed for a linear coloring can be much smaller than the treedepth of a graph. Kun et al. [5] provided a polynomial relation between the treedepth and the minimum number of colors in a linear coloring; by replacing their usage of [4] by our result (and using an improved bound for the excluded grid theorem [1]) we obtain an improved bound.

Theorem 8. There exists a polynomial $p$ such that for every integer $k$ and graph $G$, if the treedepth of $G$ is at least $k^{19} p(\log k)$, then every linear coloring of $G$ requires at least $k$ colors.

The previous bound of [5] is $k^{190} p(\log k)$.

After proving Lemma 6 in Section 2, we prove Theorem 4 in Section 3. Theorem 7 is proven in Section 3 while Theorem 8 is proven in Section 5 . The symbol $\log _{p}$ stands for base- $p \operatorname{logarithm}$ and $\log$ stands for $\log _{2}$. We denote $\varphi=\frac{1+\sqrt{5}}{2}$; note that $\varphi$ is chosen in a way so that $\varphi^{2}=\varphi+1$ and $\varphi>1$.

\section{Subcubic subtrees of trees of large treedepth}

This section focuses on proving Lemma 6 .

Schäffer [11] proved that there is a linear time algorithm for finding a vertex ranking with minimum number of colors of a tree $T$. We follow [5] for a good description of its properties.

In original Schäffer's algorithm ranks are starting from 1, however for the ease of exposition let us assume that ranks are starting from 0 . That is, the algorithm constructs a vertex ranking $\alpha: V(T) \rightarrow\{0,1,2, \ldots\}$ trying to minimize the maximum value attained by $\alpha$. Assume that $T$ is rooted in an arbitrary vertex and for every $v \in V(T)$ let $T_{v}$ be the subtree rooted at $v$.

Of central importance to Schäffer's algorithm are what we will refer to as rank lists. For a rooted tree $T$, the rank list $L(T)$ for vertex ranking $\alpha$ consists of these ranks $i$ for which there exists a path $P$ starting from the root and ending in a vertex $v$ with $\alpha(v)=i$ such that for every $u \in V(P) \backslash\{v\}$ we have $\alpha(u)<\alpha(v)$, that is, $v$ is the unique vertex of maximum rank on $P$. More formally:

- Definition 9. For a vertex ranking $\alpha$ of tree $T$, the rank list of $T$, denoted $L(T)$, can be defined recursively as $L(T)=L\left(T \backslash T_{v}\right) \cup\{\alpha(v)\}$ where $v$ is the vertex of maximum rank in $T$.

Schäffer's algorithm arbitrarily roots $T$ and builds the ranking from the leaves to the root of $T$, computing the rank of each vertex from the rank lists of each of its children. For brevity, we denote $L(v)=L\left(T_{v}\right)$ for every $v$ in $T$.

- Proposition 10. Let $\alpha$ be a vertex ranking of $T$ produced by Schäffer's algorithm and let $v \in T$ be a vertex with children $u_{1}, \ldots, u_{l}$. If $x$ is the largest integer appearing on rank lists of at least two children of $v$ (or -1 if all such rank lists are pairwise disjoint) then $\alpha(v)$ is the smallest integer satisfying $\alpha(v)>x$ and $\alpha(v) \notin \bigcup_{i=1}^{l} L\left(u_{i}\right)$.

For a node $v \in V(T)$, and vertex ranking $\alpha$, the following potential is pivotal to the analysis of Schäffer's algorithm. Let $l_{0}>l_{1}>\ldots>l_{|L(v)|-1}$ be the elements of $L(v)$ sorted in decreasing order. 


$$
\zeta(v)=\sum_{r \in L(v)} 3^{r}=\sum_{i=0}^{|L(v)|-1} 3^{l_{i}} .
$$

When we write $\zeta(T)$ for some tree $T$ we refer to $\zeta(s)$ where $s$ is a root of $T$. For our purposes, we will also use a skewed version of potential function with a different base

$$
\sigma(v)=\sum_{i=0}^{|L(v)|-1} \varphi^{l_{i}-i},
$$

where again $l_{0}>l_{1}>\ldots>l_{|L(v)|-1}$ are elements of $L(v)$ sorted in decreasing order. Throughout this section, when focusing on one node $v \in V(T)$, we use notation that $l_{i}$ is $i$-th element of set $L(v)$ when sorted in decreasing order and when 0 -based indexed.

Let us start with proving following two bounds that estimate $\operatorname{td}(T)$ in terms of $\zeta(T)$ and $\sigma(T)$.

$\triangleright$ Claim 11. $\log _{\varphi}(\sigma(T)) \geq \operatorname{td}(T)-1$.

Proof. We know that $L(T)$ is nonempty and its biggest element is equal to $\operatorname{td}(T)-1$ (we need to subtract one because we use nonnegative numbers as ranks, not positive). Therefore we have

$$
\sigma(T)=\sum_{i=0}^{|L(T)|-1} \varphi^{l_{i}-i} \geq \varphi^{l_{0}}=\varphi^{\operatorname{td}(T)-1} .
$$

Hence, $\log _{\varphi}(\sigma(T)) \geq \operatorname{td}(T)-1$, as desired.

$\triangleright$ Claim 12. $\log _{3}(\zeta(T))+\log _{3}(2)<\operatorname{td}(T)$.

Proof. We have that

$$
\begin{aligned}
\zeta(T)=\sum_{r \in L(v)} 3^{r} & \leq \sum_{r=0}^{\operatorname{td}(T)-1} 3^{r}=\frac{3^{\operatorname{td}(T)}-1}{2}, \\
2 \zeta(T) & \leq 3^{\operatorname{td}(T)}-1<3^{\operatorname{td}(T)} \\
\log _{3}(2)+\log _{3}(\zeta(T)) & <\operatorname{td}(T) .
\end{aligned}
$$

We are ready to prove Lemma 6 . Given tree $T$ we want to produce a subcubic (i.e., maximum degree at most 3) tree $S$ which is a subtree of $T$ and that fulfills $\operatorname{td}(S)>$ $\operatorname{td}(T) \log _{3}(\varphi)$.

Let us start our algorithm by arbitrary rooting $T$ and computing rank lists using Schäffer's algorithm. Then for every vertex $v \in T$ we define $C(v)$ as a set of two children of $v$ that have the biggest value of $\zeta$ in case $v$ has at least two children, or all children otherwise. Let us now define forest $F$ whose vertex set is the same as vertex set of $T$ where for every $v$ we put edges between $v$ and all elements of $C(v)$. Clearly this is a forest consisting of subcubic trees which are subtrees of $T$ (where subtree is understood as subgraph, not necessarily as some vertex $t$ along with all its descendants in a rooted tree). Let $S$ be a tree of this forest containing root of $T$. We claim that $S$ is that subcubic subtree of $T$ we are looking for. Note that computing $F$ and thus $S$ can be trivially done in polynomial time. Hence, we are left with proving that $\operatorname{td}(S)>\operatorname{td}(T) \log _{3}(\varphi)$. 
Let us root every tree of $F$ in a vertex that was closest to root of $T$ in $T$. Then compute rank lists for these trees using Schäffer's algorithm. So now, for every vertex we have two rank lists, one for $T$ and one for $F$. Let us now denote these second ranklists as $\widetilde{L}(v)$ for $v \in V(T)$ and let us define function $\widetilde{\zeta}$ which will be similar potential function as $\zeta$, but operating on rank lists $\widetilde{L}(v)$ instead of $L(v)$. Following claim will be crucial.

$\triangleright$ Claim 13. For every $v \in V(T)$ it holds that $\widetilde{\zeta}(v) \geq \sigma(v)$.

We first verify that Claim 13 implies Lemma 6 .

Proof of Lemma 6. Using also Claims 12 and 11 we infer that

$$
\begin{aligned}
& \operatorname{td}(S)>\log _{3}(\widetilde{\zeta}(S))+\log _{3}(2) \\
& \geq \log _{3}(\sigma(T))+\log _{3}(2) \\
& =\log _{\varphi}(\sigma(T)) \cdot \log _{3}(\varphi)+\log _{3}(2) \\
& \geq(\operatorname{td}(T)-1) \cdot \log _{3}(\varphi)+\log _{3}(2) \\
& =\operatorname{td}(T) \cdot \log _{3}(\varphi)-\log _{3}(\varphi)+\log _{3}(2)>\operatorname{td}(T) \cdot \log _{3}(\varphi) \text {. }
\end{aligned}
$$

Thus it remains to prove Claim 13. To this end, we prove two auxiliary inequalities.

$\triangleright$ Claim 14. For every $v \in V(T)$ it holds that $\widetilde{\zeta}(v) \geq 1+\sum_{s \in C(v)} \widetilde{\zeta}(s)$

Proof. We express every $\widetilde{\zeta}(x)$ for $x \in\{v\} \cup C(v)$ as a sum of powers of 3 and count how many times each power occurs on both sides of this claimed inequality. Consider a summand $3^{c}$. If $c>\alpha(v)$ then, by the choice of $\alpha(v), 3^{c}$ appears at most once on the right side and if it appears there, then it appears on the left side as well, so contributions of summands of form $3^{c}$ for $c>\alpha(v)$ to both sides are equal. The summand $3^{\alpha(v)}$ appears once on the left side and does not appear on the right side. For $c<\alpha(v)$, the summands of form $3^{c}$ appear at most twice in $\sum_{s \in C(v)} \widetilde{\zeta}(s)$, so their contribution to right side can be bounded from above by $\sum_{c=0}^{\alpha(v)-1} 2 \cdot 3^{c}=3^{\alpha(v)}-1$, so in fact $3^{\alpha(v)}$ from the left side contributes at least as much as remaining summands from the right side. This finishes the proof of the claim.

$\triangleright$ Claim 15. For every $v \in V(T)$ it holds that $\sigma(v) \leq 1+\sum_{s \in C(v)} \sigma(s)$

Proof. Recall that by the definition $C(v)$ is a set of two children of $v$ in $T$ with the biggest values of $\zeta$ or a set of all children of $v$ in case it has less than two of them. Observe that having bigger value of $\zeta(v)$ is another way of expressing having the set $L(v)$ bigger lexicographically when sorted in decreasing order.

If $v$ is a leaf then $C(v)$ is empty and $\sigma(v)=1$, so the inequality is obvious. Henceforth we focus on a vertex $v$ that is not a leaf. In our proof following equation will come handy:

$$
\varphi=\sum_{i=0}^{\infty} \varphi^{-2 i}
$$

It holds since $\sum_{i=0}^{\infty} \varphi^{-2 i}=\frac{1}{1-\varphi^{-2}}=\frac{\varphi^{2}}{\varphi^{2}-1}=\frac{\varphi^{2}}{\varphi}=\varphi$.

Let us now analyze $L(v)$. It consists of some prefix $P$ of values that appeared exactly once in children of $v$, then $\alpha(v)$ and then nothing (when enumerated from the biggest to the smallest). Let us now denote by $A_{i}$ intersection of $L\left(u_{i}\right)$ and $P$, where $u_{i}$ is $i$-th child of $v$ when sorted in nonincreasing order by their values $\zeta\left(u_{i}\right)$ (1-based). We distinguish two cases: 
Case 1: $\boldsymbol{A}_{2}$ is nonempty. If $A_{2}$ is nonempty then in particular it means that $v$ has at least two children. Let us denote the biggest element of $L\left(u_{2}\right)$ by $d$. We have that $d \in P$, but $d$ is not the biggest element of $P$. Its contribution to $\sigma\left(u_{2}\right)$ is $\varphi^{d}$, however its contribution to $\sigma(v)$ is at most $\varphi^{d-1}$ (because of the skew and since $d$ is not the biggest element of $P)$. Contribution to $\sigma(v)$ of elements smaller than $d$ can be bounded from above by $\varphi^{d-3}+\varphi^{d-5}+\ldots$ We know that $d=l_{j}$ for some $j$, where $j \geq 1$ and $L(v)$ consists of elements $l_{0}>l_{1}>\ldots>l_{|L(v)-1|}$. We have that $l_{k} \in L\left(u_{1}\right)$ for $k<j$ and that $l_{j} \geq l_{i}+(i-j)$ for $i \geq j$, so $l_{i}-i \leq l_{j}-j-2(i-j)=d-j-2(i-j)$.

We can deduce that

$$
\begin{aligned}
\sigma(v) & =\sum_{i=0}^{|L(v)|-1} \varphi^{l_{i}-i}=\sum_{i=0}^{j-1} \varphi^{l_{i}-i}+\sum_{i=j}^{|L(v)|-1} \varphi^{l_{i}-i} \leq \sigma\left(u_{1}\right)+\sum_{i=j}^{|L(v)|-1} \varphi^{d-j-2(i-j)} \\
& \leq \sigma\left(u_{1}\right)+\varphi^{d-j} \sum_{i=j}^{\infty} \varphi^{-2(i-j)}=\sigma\left(u_{1}\right)+\varphi^{d-j} \sum_{i=0}^{\infty} \varphi^{-2 i}=\sigma\left(u_{1}\right)+\varphi^{d-j+1} \\
& \leq \sigma\left(u_{1}\right)+\varphi^{d} \leq \sigma\left(u_{1}\right)+\sigma\left(u_{2}\right)<1+\sigma\left(u_{1}\right)+\sigma\left(u_{2}\right)
\end{aligned}
$$

which is what we wanted to prove.

Case 2: $\boldsymbol{A}_{2}$ is empty. Let us now introduce a few variables:

- $d$ - the biggest integer number smaller than $\alpha(v)$ that is not an element of $L\left(u_{1}\right)$.

We know that elements from $d+1$ to $\alpha(v)-1$ belong to $L\left(u_{1}\right)$.

- $k$ - shorthand for number of these elements (which is equal to $\alpha(v)-1-d$ ).

$k$ can be zero, but cannot be negative.

- $g$ - the number of elements of $L(v)$ that are bigger than $\alpha(v)$.

Then from the definition of $\alpha(v)$ either

- $d=-1$; or

- $v$ has at least two children and $L\left(u_{2}\right)$ contains a number that is at least $d$.

Because of that we have $1+\sum_{s \in C(v)} \sigma(s) \geq \sigma\left(u_{1}\right)+\varphi^{d}$. We know that $\sum_{s \in C(v)}$ is either $\sigma\left(u_{1}\right)$ or $\sigma\left(u_{1}\right)+\sigma\left(u_{2}\right)$, depending on whether $v$ has only one child or more. If $d=-1$ then $1 \geq \varphi^{d}$ and stated inequality holds. If $d \neq-1$ then $u_{2}$ exists and $\sigma\left(u_{2}\right) \geq \varphi^{d}$.

Note that either $k>0$ or $g>0$, because if $k=g=0$ then $d=\alpha(v)-1$ and $L\left(u_{1}\right)$ cannot contain elements bigger then $\alpha(v)$ (because $g=0$ ), cannot contain $\alpha(v)$ (from the definition of $\alpha(v)$ ) and cannot contain $\alpha(v)-1$ (since $d=\alpha(v)-1$ ), so its biggest element is at most $d-1$. If $d=-1$ then it means that $v$ is a leaf, but we already assumed it is not one. However, if $v$ has at least two children and $L\left(u_{2}\right)$ contains a number that is at least $d$, then it contradicts the assumption that $\zeta\left(u_{1}\right) \geq \zeta\left(u_{2}\right)$. So indeed it holds that $k>0$ or $g>0$ and therefore $k+g \geq 1$.

We have that

$$
\sigma(v)-\sigma\left(u_{1}\right) \leq \varphi^{\alpha(v)-g}-\left(\varphi^{\alpha(v)-g-1}+\varphi^{\alpha(v)-g-3}+\ldots+\varphi^{\alpha(v)-g-2 k+1}\right),
$$

which is because summands coming from numbers bigger than $\alpha(v)$ in $L(v)$ and $L\left(u_{1}\right)$ cancel out $\left(A_{2}\right.$ is empty, so all elements of $L(v)$ different than $\alpha(v)$ come from $\left.L\left(u_{1}\right)\right)$ and new rank $\alpha(v)$ contributes $\varphi^{\alpha(v)-g}$ to $\sigma(v)$ whereas $L\left(u_{1}\right)$ contains numbers from $d+1$ up to $\alpha(v)-1$ and their contribution to $\sigma\left(u_{1}\right)$ is $\varphi^{\alpha(v)-g-1}+\varphi^{\alpha(v)-g-3}+\ldots+\varphi^{\alpha(v)-g-2 k+1}$. We conclude that $\sigma(v)-\sigma\left(u_{1}\right) \leq \varphi^{-g}\left(\varphi^{\alpha(v)}-\left(\varphi^{\alpha(v)-1}+\varphi^{\alpha(v)-3}+\ldots+\varphi^{\alpha(v)-2 k+1}\right)\right)$. On the other hand since $\varphi^{2}=\varphi+1$ we have that

$$
\begin{aligned}
& \varphi^{\alpha(v)}=\varphi^{\alpha(v)-1}+\varphi^{\alpha(v)-2}=\varphi^{\alpha(v)-1}+\varphi^{\alpha(v)-3}+\varphi^{\alpha(v)-4}=\ldots= \\
& =\left(\varphi^{\alpha(v)-1}+\varphi^{\alpha(v)-3}+\ldots+\varphi^{\alpha(v)-2 k+1}\right)+\varphi^{\alpha(v)-2 k} .
\end{aligned}
$$


Because of that we have

$$
\sigma(v)-\sigma\left(u_{1}\right) \leq \varphi^{-g} \cdot \varphi^{\alpha(v)-2 k}=\varphi^{\alpha(v)-2 k-g}=\varphi^{\alpha(v)-(\alpha(v)-1-d)-k-g}=\varphi^{d+1-(k+g)} \leq \varphi^{d} .
$$

From that we conclude that $\sigma(v) \leq \sigma\left(u_{1}\right)+\varphi^{d} \leq 1+\sum_{s \in C(v)} \sigma(s)$, what concludes proof of this claim.

Now, having claims 15 and 14 proven, we can wrap our reasoning up. If $v$ is a leaf then $\sigma(v)=\widetilde{\zeta}(v)=1$. If $v$ is not a leaf then we know that $\sigma(v) \leq 1+\sum_{s \in C(v)} \sigma(s)$ and $\widetilde{\zeta}(v) \geq 1+\sum_{s \in C(v)} \widetilde{\zeta}(s)$, so by straightforward induction we get that $\sigma(v) \leq \widetilde{\zeta}(v)$ for every $v \in V(T)$, as desired by Claim 13 .

\section{Proof of Theorem 4}

Let $G$ be a nonempty graph and let $r=\operatorname{td}(G) /(\operatorname{tw}(G)+1)$. Recall that our goal is to show existence of a subcubic tree $H$ being a subgraph of $G$ such that $\operatorname{td}(H)$ is $\Omega(r / \log (\operatorname{tw}(G)+1))$. Without loss of generality we may assume that $G$ is connected, as otherwise we focus on the connected component of $G$ of maximum treedepth. Also, the statement is trivial for $\operatorname{tw}(G) \leq 1$ (when $G$ is a tree) and when $r \leq 2$, so assume otherwise.

We consider a greedy tree decomposition of $G$, as defined in [4]. A greedy tree decomposition is a tree decomposition that can be also interpreted as a treedepth decomposition.

Recall that a tree decomposition of a graph $G$ is a pair $(T, \beta)$ where $T$ is a rooted tree and $\beta: V(T) \rightarrow 2^{V(G)}$ is such that for every $v \in V(G)$ the set $\{t \in V(T) \mid v \in \beta(t)\}$ induces a connected nonempty subtree of $T$ and for every $u v \in E(G)$ there exists $t \in V(T)$ with $u, v \in \beta(t)$. A tree decomposition $(T, \beta)$ of a graph $G$ is greedy if

1. $V(T)=V(G)$,

2. for every $u v \in E(G)$, the nodes $u$ and $v$ in $T$ are in ancestor-descendant relation in $T$, and

3. for every vertex $u \in V(T)$ and its child $v$ there is some descendant $w$ of $v$ in $T$ such that $u w \in E(G)$.

Lemma 3.6. in [4] states that for every connected graph $G$ there exists a greedy tree decomposition $(T, \beta)$ of width $\operatorname{tw}(G)$, that is, all bags $\beta(t)$ for $t \in V(T)$ have size bounded by $\operatorname{tw}(G)+1$. Let $(T, \beta)$ be such a decomposition of our graph $G$. By Lemma 1 we get that $\operatorname{td}(T) \geq r$, as otherwise we would be able to construct treedepth decomposition of too low treedepth.

In the remainder of the proof we show the following lemma.

- Lemma 16. Let $G$ be a connected graph, $(T, \beta)$ be a greedy tree decomposition of $G$, and let $\tau \geq 2$ be such that $|\beta(t)| \leq \tau$ for every $t \in V(T)$. Then $G$ contains a subcubic tree of treedepth $\Omega(\operatorname{td}(T) / \log \tau)$.

Theorem 4 follows immediately from Lemma 16 applied to the tree decomposition $(T, \beta)$ of $G$. Thus, it remains to prove Lemma 16.

To this end, we first apply Lemma 6 to tree $T$ and obtain a subcubic tree $S$ such that

$$
\operatorname{td}(S) \geq r \cdot \log _{3}(\varphi)
$$

Second, we apply the core part of the reasoning of Kawarabayashi and Rossman [4]. The construction of Section 5 of [4] can be encapsulated in the following lemma. 
- Lemma 17 (Section 5 of [4]). Let $(T, \beta)$ be a greedy tree decomposition of graph $G$ and let $\tau=\max _{t \in V(T)}|\beta(t)|$. Then for every subcubic subtree $S$ of $T$ there exists a subtree $F$ of $G$ such that $V(S) \subseteq V(F)$ and the maximum degree of $F$ is bounded by $\tau+2$.

By application of Lemma 17 to our decomposition $(T, \beta)$ and subtree $S$ we get a tree $F$ in $G$, which has large treedepth, as we show in a moment. To this end, we need the following simple bound on treedepth of trees.

- Lemma 18. For every tree $H$ with maximum degree bounded by $d \geq 2$ it holds that

$$
\log _{d}|V(H)| \leq \operatorname{td}(T) \leq 1+\log _{2}|V(H)| .
$$

Proof. We use the following equivalent recursive definition of treedepth: Treedepth of an empty graph is 0, treedepth of a disconnected graph equals the maximum of treedepth over its connected components, while for nonempty connected graphs $G$ we have $\operatorname{td}(G)=$ $1+\min _{v \in V(G)} \operatorname{td}(G-v)$.

For the lower bound, for $k \geq 1$ let $f_{d}(k)$ be the maximum possible number of vertices of a tree of maximum degree at most $d$ and treedepth at most $k$. Clearly, $f_{d}(1)=1$. Since removing a single vertex from a tree of maximum degree at most $d$ results in at most $d$ connected components, we have that

$$
f_{d}(k+1) \leq 1+d \cdot f_{d}(k)
$$

Consequently, we obtain by induction that

$$
f_{d}(k) \leq d^{k}-1
$$

This proves the lower bound. For the upper bound, note that in every tree $T$ there exists a vertex $v \in V(T)$ such that every connected component of $T-\{v\}$ has at most $|V(T)| / 2$ vertices. Consequently, if we define $g(n)$ to be the maximum possible treedepth of an $n$-vertex tree, then $g(1)=1$ and we have that

$$
g(n) \leq 1+g(\lfloor n / 2\rfloor) .
$$

This proves the upper bound.

By (1) and Lemma 18 we get that $|V(S)| \geq 2^{r \cdot \log _{3}(\varphi)-1}$. This implies that also

$$
|V(F)| \geq 2^{r \cdot \log _{3}(\varphi)-1} .
$$

As $S$ is subcubic, by Lemma 17 we know that the maximum degree of $F$ is bounded by $\operatorname{tw}(G)+3$. Therefore Lemma 18 and (2) jointly imply that

$$
\operatorname{td}(F) \geq \log _{(\operatorname{tw}(G)+3)} 2^{r \cdot \log _{3}(\varphi)-1} \geq \frac{r \cdot \log _{3}(\varphi)-1}{\log (\operatorname{tw}(G)+3)} \geq \frac{\log _{3}(\varphi)}{20} \cdot r / \log (\operatorname{tw}(G)+1) .
$$

Here, the last inequality follows from the assumptions $r>2$ and $\operatorname{tw}(G) \geq 2$; note that the constant 20 is sufficiently large constant for the estimations to work.

As tree $F$ is not necessarily subcubic, we apply one more time Lemma 6 and get a subcubic subtree $H$ of $F$ such that

$$
\operatorname{td}(H) \geq \operatorname{td}(F) \cdot \log _{3}(\varphi) \geq \frac{\log _{3}(\varphi)^{2}}{20} \cdot r / \log (\operatorname{tw}(G)+1),
$$

which finishes the proof of Lemma 16 and of Theorem 4. 


\section{Proof of Theorem 7}

Proof of Theorem 7. Without loss of generality we can assume that the input graph $G$ is connected. As in the proof of Lemma 2, we apply the polynomial-time approximation algorithm for treewidth [3], to compute a tree decomposition $\left(T_{0}, \beta_{0}\right)$ of $G$ with $\mathcal{O}(n)$ nodes of $T_{0}$ and $|\beta(t)| \leq \tau$ for every $t \in V\left(T_{0}\right)$ and some $\tau=\mathcal{O}(\operatorname{tw}(G) \sqrt{\log \operatorname{tw}(G)})$. As discussed in [4], one can in polynomial time turn $\left(T_{0}, \beta_{0}\right)$ into a greedy tree decomposition $(T, \beta)$ of $G$ without increasing the maximum size of a bag, that is, still $|\beta(t)| \leq \tau$ for every $t \in V(T)$. We apply Lemma 1 to $(T, \beta)$, returning a treedepth decomposition of $G$ of width at most $\tau \cdot \operatorname{td}(T)=\mathcal{O}(\operatorname{td}(T) \operatorname{tw}(G) \sqrt{\log \operatorname{tw}(G)})$.

It remains to bound $\operatorname{td}(T)$. Lemma 16 asserts that $G$ contains a subcubic tree $H$ of treedepth $\Omega(\operatorname{td}(T) / \log \tau)$. Therefore $\operatorname{td}(T)=\mathcal{O}(\operatorname{td}(H) \log \tau)=\mathcal{O}(\operatorname{td}(G) \log \operatorname{tw}(G))$ and thus the width of the computed treedepth decomposition is $\mathcal{O}\left(\operatorname{td}(G) \operatorname{tw}(G) \log ^{3 / 2} \operatorname{tw}(G)\right)$. This finishes the proof of Theorem 7.

\section{$5 \quad$ Proof of Theorem 8}

Here we show how to assemble the proof of Theorem 8 from Theorem 4, a number of intermediate results of [5], and an improved excluded grid theorem due to Chuzhoy and Tan [1]:

- Theorem 19 ([1]). There exists a polynomial $p_{\mathrm{GMT}}$ such that for every integer $k$ if a graph $G$ has treewidth at least $k^{9} p_{\mathrm{GMT}}(\log k)$ then $G$ contains a $k \times k$ grid as a minor.

The following two results were proven in [5].

- Lemma 20 ([5]). If a graph $G$ contains a $k \times k$ grid as a minor, then every linear coloring of $G$ requires $\Omega(\sqrt{k})$ colors.

- Lemma 21 ([5]). If $G$ is a tree of treedepth $d$ and maximum degree $\Delta$, then every linear coloring of $G$ requires at least $d / \log _{2}(\Delta)$ colors.

Recall that Theorem 4 asserts that there exists a constant $C$ such that for every graph $G$ and integers $a, b \geq 2$, if the treedepth of $G$ is at least $C a b \log a$, then either the treewidth of $G$ is at least $a$ or $G$ contains a subcubic tree of treedepth at least $b$. Applying this theorem to $a=\theta\left(k^{2}\right)$ and $b=k \log _{2}(3)$, one obtains that if the treedepth of $G$ is $\Omega\left(k^{19} p_{\mathrm{GMT}}(\log k) \log k\right)$, then $G$ contains either a $\theta\left(k^{2}\right) \times \theta\left(k^{2}\right)$ grid minor or a subcubic tree of treedepth at least $k \log _{2}(3)$. In the first outcome, Lemma 20 gives the desired number of colors of a linear coloring, while in the second outcome the same result is obtained from Lemma 21. This concludes the proof of Theorem 8 .

\section{An example of a tree with treedepth quadratic in the height of the binary tree or logarithm of a length of a path}

In this section we provide a construction of a family of trees $\left(G_{n}\right)_{n \geq 1}$ such that

1. The tree $G_{n}$ does not contain a path with $2^{n+2}$ vertices.

2. The tree $G_{n}$ does not contain a subdivision of a full binary tree of depth $n+2$.

3. The treedepth of $G_{n}$ is at least $\left(\begin{array}{c}n+1 \\ 2\end{array}\right)$.

We will consider each tree $G_{n}$ as a rooted tree. The tree $G_{1}$ consists of a single vertex. For $n \geq 2$, the tree $G_{n}$ is defined recursively as follows. We take a path $P_{n}$ with $2^{n}$ vertices and for each $v \in V\left(P_{n}\right)$ we create a copy $C_{n}^{v}$ of $G_{n-1}$ and attach its root to $v$. We root $G_{n}$ in one of the endpoints of $P_{n}$; see Figure 1. We now proceed with the proof of the properties of $G_{n}$. 


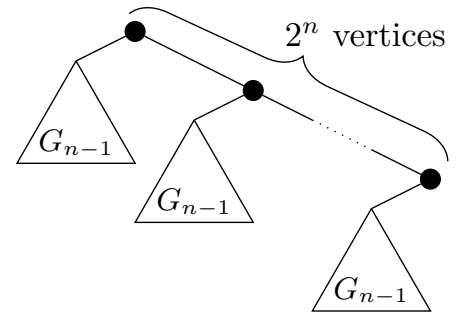

Figure 1 Construction of $G_{n}$.

Since every path in $G_{n}$ is contained in at most two root-to-leaf paths (not necessarily edge-disjoint), to show Property (1) it suffices to show the following.

- Lemma 22. Every root-to-leaf path in $G_{n}$ contains less than $2^{n+1}$ vertices.

Proof. We prove the statement by induction on $n$. For $n=1$ the statement is straightforward. For the inductive step, observe that every root-to-leaf path in $G_{n}$ consists of a subpath of $P_{n}$ (which has $2^{n}$ vertices) and a root-to-leaf path in one of the copies $C_{n}^{v}$ of $G_{n-1}$ (which has less than $2^{n}$ vertices by the inductive assumption).

We say that a subtree $H$ of $G_{n}$ that is a subdivision of a full binary tree of height $h \geq 1$ is aligned if $h=1$ or $h \geq 2$ and the closest to the root vertex of $H$ is of degree 2 in $H$ and its deletion breaks $H$ into two subtrees containing a subdivision of a full binary tree of height $h-1$. In other words, an aligned subtree has the same ancestor-descendant relation as the tree $G_{n}$. Observe that any subtree $H_{0}$ of $G_{n}$ that is a subdivision of a full binary tree of height $h \geq 2$ contains a subtree that is an aligned subdivision of a full binary tree of height $h-1$. Therefore, to prove Property (2), it suffices to show the following.

- Lemma 23. $G_{n}$ does not contain an aligned subdivision of a full binary tree of height $n+1$.

Proof. We prove the claim by induction on $n$. It is straightforward for $n=1$. For $n \geq 2$, let $H$ be such an aligned subtree of $G_{n}$ and let $w$ be the closest to the root of $G_{n}$ vertex of $H$. If $w \in V\left(C_{n}^{v}\right)$ for some $v \in V\left(P_{n}\right)$, then $H$ is completely contained in $C_{n}^{v}$, which is a copy of $G_{n-1}$. Otherwise, $w \in V\left(P_{n}\right)$ and thus one of the components of $H-\{w\}$ lies in $C_{n}^{w}$. However, this component contains an aligned subdivision of a full binary tree of height $n$. In both cases, we obtain a contradiction with the inductive assumption.

We are left with the treedepth lower bound of Property (3). To this end, we consider the following families of trees. For integers $a, b \geq 1$, the family $\mathcal{G}_{a, b}$ contains all trees $H$ that are constructed from a path $P_{H}$ with at least $2^{a}$ vertices by attaching, for every $v \in V\left(P_{H}\right)$, a tree $T_{v}$ of treedepth at least $b$ by an edge to $v$. We show the following.

Lemma 24. For every $H \in \mathcal{G}_{a, b}$ we have $\operatorname{td}(H) \geq a+b$.

Proof. We prove the lemma by induction on $a$. For $a=1$ we have $\operatorname{td}(H) \geq a+1$ and $H$ contains two vertex-disjoint subtrees of treedepth at least $b$ each. Assume then $a>1$ and $H \in \mathcal{G}_{a, b}$. Then for every $v \in V(H), H-v$ contains a connected component that contains a subtree belonging to $\mathcal{G}_{a-1, b}$. This finishes the proof. 
We show Property (3) by induction on $n$. Clearly, $\operatorname{td}\left(G_{1}\right)=1=\left(\begin{array}{c}1+1 \\ 2\end{array}\right)$. Consider $n \geq 2$. Since the treedepth of $G_{n-1}$ is at least $\left(\begin{array}{l}n \\ 2\end{array}\right)$, we have that $G_{n} \in \mathcal{G}_{n,\left(\begin{array}{l}n \\ 2\end{array}\right)}$. By Lemma 24, we have that

$$
\operatorname{td}\left(G_{n}\right) \geq n+\left(\begin{array}{l}
n \\
2
\end{array}\right)=\left(\begin{array}{c}
n+1 \\
2
\end{array}\right)
$$

This finishes the proof of Property (3).

\section{Conclusions}

We have provided improved bounds for the excluded minor approximation of treedepth of Kawarabayashi and Rossman [4]. Our main result, Theorem 4, is close to being optimal in the following sense: as witnessed by the family of trees, if one considers the measure $r:=\operatorname{td}(G) / \operatorname{tw}(G)$, one cannot hope to find a tree in $G$ of treedepth larger than $r$. We pose getting rid of the $\log (\operatorname{tw}(G)+1)$ factor in Theorem 4 as an open problem. Improving the $C k^{3} \log k$ bound of Corollary 5 to $C k^{3-\varepsilon}$ for some $\varepsilon>0$ seems much more challenging.

Our main result can be applied to a polynomial-time treedepth approximation algorithm, improving upon state-of-the-art tradeoff trick. As a second open problem, we ask for a polynomial-time or single-exponential in treedepth parameterized algorithm for constant or polylogarithmic approximation of treedepth.

\section{References}

1 Julia Chuzhoy and Zihan Tan. Towards Tight(er) Bounds for the Excluded Grid Theorem. In Timothy M. Chan, editor, Proceedings of the Thirtieth Annual ACM-SIAM Symposium on Discrete Algorithms, SODA 2019, San Diego, California, USA, January 6-9, 2019, pages 1445-1464. SIAM, 2019. doi:10.1137/1.9781611975482.88.

2 Zdenek Dvorak, Archontia C. Giannopoulou, and Dimitrios M. Thilikos. Forbidden graphs for tree-depth. Eur. J. Comb., 33(5):969-979, 2012. doi:10.1016/j.ejc.2011.09.014.

3 Uriel Feige, MohammadTaghi Hajiaghayi, and James R. Lee. Improved Approximation Algorithms for Minimum Weight Vertex Separators. SIAM J. Comput., 38(2):629-657, 2008. doi: $10.1137 / 05064299 \mathrm{X}$

4 Ken-ichi Kawarabayashi and Benjamin Rossman. A Polynomial Excluded-Minor Approximation of Treedepth. In Artur Czumaj, editor, Proceedings of the Twenty-Ninth Annual ACM-SIAM Symposium on Discrete Algorithms, SODA 2018, New Orleans, LA, USA, January 7-10, 2018, pages 234-246. SIAM, 2018. doi:10.1137/1.9781611975031.17.

5 Jeremy Kun, Michael P. O'Brien, and Blair D. Sullivan. Treedepth Bounds in Linear Colorings. CoRR, abs/1802.09665, 2018. arXiv: 1802.09665

6 Jaroslav Nesetril and Patrice Ossona de Mendez. Tree-depth, subgraph coloring and homomorphism bounds. Eur. J. Comb., 27(6):1022-1041, 2006. doi:10.1016/j.ejc.2005.01.010.

7 Jaroslav Nesetril and Patrice Ossona de Mendez. Sparsity - Graphs, Structures, and Algorithms, volume 28 of Algorithms and combinatorics. Springer, 2012. doi:10.1007/ 978-3-642-27875-4.

8 Jaroslav Nesetril and Patrice Ossona de Mendez. On Low Tree-Depth Decompositions. Graphs and Combinatorics, 31(6):1941-1963, 2015. doi:10.1007/s00373-015-1569-7.

9 Felix Reidl, Peter Rossmanith, Fernando Sánchez Villaamil, and Somnath Sikdar. A Faster Parameterized Algorithm for Treedepth. In Javier Esparza, Pierre Fraigniaud, Thore Husfeldt, and Elias Koutsoupias, editors, Automata, Languages, and Programming - 41st International Colloquium, ICALP 2014, Copenhagen, Denmark, July 8-11, 2014, Proceedings, Part I, volume 8572 of Lecture Notes in Computer Science, pages 931-942. Springer, 2014. doi: 10.1007/978-3-662-43948-7_77. 
10 Neil Robertson and Paul D. Seymour. Graph minors. II. Algorithmic aspects of tree-width. Journal of Algorithms, 7(3):309-322, 1986.

11 Alejandro A. Schäffer. Optimal Node Ranking of Trees in Linear Time. Inf. Process. Lett., 33(2):91-96, 1989. doi:10.1016/0020-0190 (89) 90161-0. 